\title{
Landmine injuries in Eritrea
}

\author{
Kurt Hanevik, Gunnar Kvåle
}

During the 29 year guerrilla war against the Ethiopians, 1-2 million landmines were scattered over much of Eritrea. ${ }^{1}$ We aimed to assess the incidence of civilian landmine injuries over time since the war ended in May 1991 and to evaluate the sex, age, and pattern of injuries of landmine victims.

\section{Subjects, methods, and results}

Data were collected from operation room records for patients at hospitals in Mendefera, Keren, Naqfa, and Afabet in 1995. These were the only places where surgery was offered to civilians during this time in the provinces of Seraye, Senhit, and Sahel, which had a population of about 759000 . The study covers 248 civilians injured by landmines between June 1991 and March 1995.

For the incidence pattern over time, only the patients from Senhit (total population 275000 ) treated at Keren hospital $(n=159)$ were included, because records were continuous and more reliable there. For this area, the mean annual incidence per 100000 population was 136.7 in June-August 1991, 26.2 in September-December 1991, 10.2 in January-June 1992, and 8.0 in June-December 1992.

The mean age of the patients injured by landmines was 20.8 years. Of the 248 mine victims who had surgical records, $223(90 \%)$ were male, $102(41 \%)$ were aged under 15, and 54 (22\%) were 15-19 years old. Sex difference increased with increasing age.

The pattern of injury classification developed by Coupland and Korver was used (pattern 1: severe damage of a foot or leg, often leading to amputation; pattern 2: multilocular wounds from penetrating fragments; pattern 3: upper limb and face, eye, and chest injuries). ${ }^{2}$ Pattern 3 was the most common type of injury $(118(48 \%))$, followed by pattern $1(65(26 \%))$; pattern 2 was found in $42(17 \%)$ of patients, and 23 (9\%) had ambiguous patterns.

A total of $126(51 \%)$ of the patients had amputations-51 patients (21\%) needed leg amputations, $24(10 \%)$ had a hand or arm amputated, and 51 $(21 \%)$ had finger amputations. The rest received treatment such as debridement.

After the war ended in May 1991 the number of patients injured by mines rose (figure), reaching a peak in the second month, after which it declined and stabilised for a year. In each of the following years only four or five people injured by landmines were seen at Keren hospital, typically during the summer when mines are washed up or moved by heavy rainfall.

\section{Comment}

People need to be made rapidly aware of mines after conflict, and movement needs to be restricted before operations to clear mines begin. The high number of landmine injuries just after liberation might be explained by people's freedom to move into areas that had been inaccessible because of warfare and that

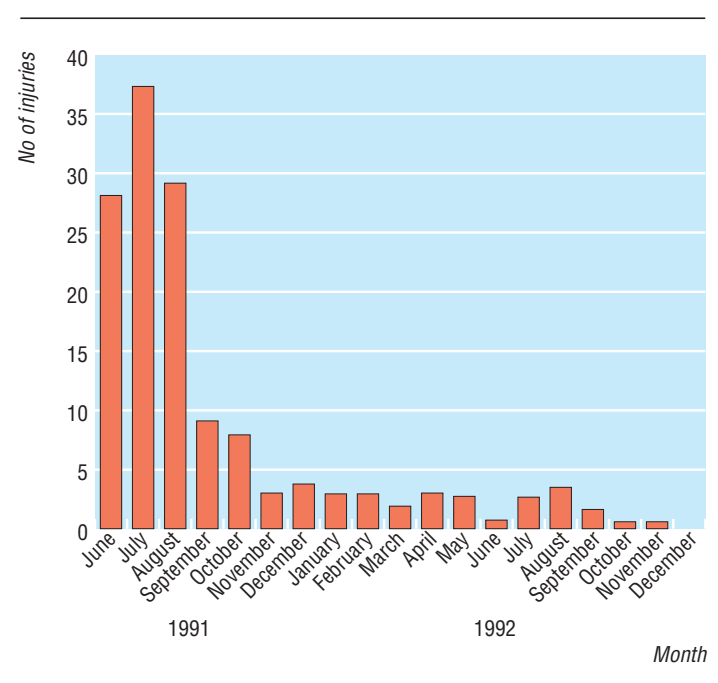

Centre for

International

Health, University

of Bergen, N-5021

Bergen, Norway

Kurt Hanevik

research fellow

Gunnar Kvåle

professor

Correspondence to:

K Hanevik

kurt.hanevik@ikb.

uib.no

BMJ 2000;321:1189

Number of civilian landmine injuries treated at Keren hospital,

Eritrea, June 1991-December 1992

were believed to be safe after liberation. There are several reasons for the observed decrease in injuries from mines. By seeing other people and livestock get hurt or killed, people have learned what areas to avoid. Also, although intensive clearance of mines was done in the three years after liberation, because of poor equipment and technique many mines remained. ${ }^{1}$

The high percentage of injuries to the upper body shows that people need to be taught about the dangers of picking up any suspect objects.

In comparison with other studies, ${ }^{2-4}$ we found that a higher percentage of children are injured by landmines in Eritrea than elsewhere, possibly because Eritrean boys start herding at a rather young age.

Ethiopia and Eritrea again went to war against each other in 1998 and there have been allegations of new mines being laid. International pressure is needed to stop this new manmade disaster from creating yet more suffering and disability.

Contributors: $\mathrm{KH}$ had the initial idea and did the data collection in Eritrea. GK was involved in organising and analysing data and in writing the paper. $\mathrm{KH}$ is the guarantor.

Funding: None.

Competing interests: None declared.

1 Taylor C. Landmines warfare-mines and engineer munitions in Eritrea Report NGIC-1166-004-94. Charlottesville, VA: National Ground Intelligence Center, 1994.

2 Coupland RM, Korver A. Injuries from anti-personnel mines: the experience of the International Committee of the Red Cross. BMJ 1991;303:1509-12

3 Ascherio A, Biellik R, Epstein A, Snetro G, Gloyd S, Ayotte B, et al. Deaths and injuries caused by land mines in Mozambique. Lancet 1995;346: $721-4$.

4 Anderson N, Palha da Sousa C, Paredes S. Social costs of land mines in four countries: Afghanistan, Bosnia, Cambodia, and Mozambique. BMJ 1995;311:718-21.

(Accepted 10 July 2000) 